\title{
Chromatic Polynomial and Chromatic Uniqueness of Necklace Graph
}

\author{
Usman Ali, Syed Ahtsham Ul Haq Bokhary, Sakina Ashraf \\ Centre for Advanced Studies in Pure and Applied Mathematics, \\ Bahauddin Zakariya University, Multan, Pakistan \\ E-mail: uali@bzu.edu.pk, sihtsham@gmail.com, sheeba_dgk@yahoo.com \\ Mathematics Subject Classification: 05C15, 05C65
}

\begin{abstract}
For a graph $G$, let $P(G, \lambda)$ be its chromatic polynomial. Two graphs $G$ and $H$ are said to be chromatically equivalent if $P(G, \lambda)=$ $P(H, \lambda)$. A graph is said to be chromatically unique if no other graph shares its chromatic polynomial. In this paper, chromatic polynomial of the necklace graph $N_{n}$, for $n \geq 2$ has been determined. It is further shown that $N_{3}$ is chromatically unique.
\end{abstract}

Keywords: chromatic polynomial, chromatically equivalent, chromatically unique, necklace graph

\section{Introduction}

A $k$-coloring of a graph $G$ is a mapping $\phi: V(G) \rightarrow\{1,2,3, \ldots, k\}$ such that $\phi(m) \neq \phi(n)$ for any edge $e=m n$. A minimum number $k$ such that $G$ has a proper coloring is called chromatic number, and $G$ is called $k$-colorable graph. Many useful tools had been developed for solving graph coloring problems. Birkhoff [1] was the first who proposed a method to determine $k$ colorable graphs by introducing a function $P(G, \lambda)$ that represent the number of all proper coloring of a graph $G$ with $k$ colors. $P(G, \lambda)$ is called chromatic polynomial of $G$. 
Chao and Whithead Jr. [2] defined a graph to be chromatically unique if no other graphs share its chromatic polynomial and the two graphs are chromatically equivalent if they share a same chromatic polynomial.

Let $P_{n}$ be a path of length $n-1$ defined on the $n$ vertices $a_{1} \ldots a_{n}$. The comb graph denoted by $C b_{n}$ is the tree graph consisting of a path $P_{n}$ together with vertices $b_{0}, b_{1}, \ldots, b_{n+1}$ and edges $b_{i} a_{i}$, with $0 \leq i \leq n$. The necklace graph $N_{n}$ is a 3- regular Halin graph [6] and is obtained from comb graph $C b_{n}$ by adding the edges $a_{1} b_{0}, b_{0} b_{n+1}, b_{n+1} b_{n}$ and $b_{i} b_{i+1}$, with $1 \leq i \leq n-1$. Alternatively, vertex and edge set of $N_{n}$ is defined as:

$$
\begin{gathered}
V\left(N_{n}\right)=V\left(C b_{n}\right)=\left\{a_{1}, a_{2}, \ldots a_{n}, b_{0}, b_{1}, \ldots b_{n+1}\right\} \\
E\left(N_{n}\right)=\left\{a_{i} a_{i+1}, a_{i} b_{i}, b_{i} b_{i+1}: 1 \leq i \leq n-1\right\} \cup\left\{b_{0} b_{1}, b_{0} a_{1}, b_{n} b_{n+1}, a_{n} b_{n+1}\right\} .
\end{gathered}
$$

The vertices $b_{0}$ and $b_{n+1}$ are called end vertices of the Necklace graph. The $N_{n}$ has $2 n+2$ vertices and $3 n+3$ edges. It is important to note that the necklace graph $N_{n}$ is a 2-connected 3-regular graph (see Figure 1).

The main objective of this paper is to study the chromatic polynomial and

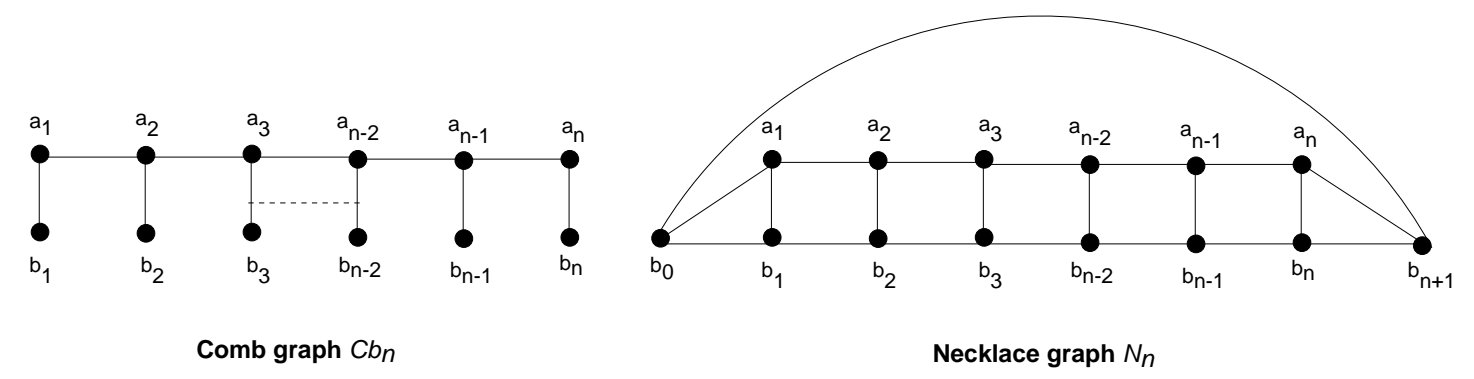

Figure 1: Necklace Graph

chromatic uniqueness of necklace graph $N_{n}$.

\section{Basic Results}

In this section, some of the known results are stated that help in proving the main results of this paper.

Theorem 2.1 (Fundamental reduction theorem (FRT) [9]) Let G be a graph and $e$ an edge of $G$. Then

$$
P(G)=P(G-e)-P(G . e)
$$


where $G-e$ is the graph obtained from $G$ by deleting e, and $G$. e is the graph obtained from $G$ by contracting the end vertices of e and removing all but one of the multiple edges, if they arise.

Theorem 2.2 [4]) Let $G$ be a graph with $n$ vertices and $m$ edges then

$$
P(G, x)=\sum_{i=0}^{n} a_{i} x^{n-i},
$$

is a polynomial in $x$ of degree $n$ such that

1. The coefficient $a_{i}$ are integers and alternate in sign;

2. $a_{0}=1, a_{n}=0, a_{1}=-m$;

3. $a_{2}=\left(\begin{array}{c}m \\ 2\end{array}\right)-t_{1}(G), a_{3}=-\left(\begin{array}{c}m \\ 3\end{array}\right)+(m-2) t_{1}(G)+t_{2}(G)-2 t_{3}(G)$;

4. $a_{4}=\left(\begin{array}{c}m \\ 4\end{array}\right)-\left(\begin{array}{c}m-2 \\ 2\end{array}\right) t_{1}(G)-\left(\begin{array}{c}t_{1}(G) \\ 2\end{array}\right)-(m-3) t_{2}(G)$

$+(2 m-9) t_{3}(G)-t_{4}(G)-6 t_{5}(G)+t_{6}(G)+2 t_{7}(G)+3 t_{8}(G)$.

$t_{1}(G)$ : the number of $C_{3}$.

$t_{2}(G)$ : the number of pure $C_{4}$.

$t_{3}(G)$ : the number of $K_{4}$.

$t_{4}(G)$ : the number of pure pentagons $C_{5}$.

$t_{5}(G)$ : the number of $K_{5}$ subgraph.

$t_{6}(G)$ : the number of $2-3$ complete bipartite graphs.

$t_{7}(G)$ : the number of 5-vertex wheels with one spoke deleted.

$t_{8}(G)$ : the number of wheel $W_{5}$.

Theorem 2.3 [8] let $G$ be a graph of order $n$ and size $m$. Then

$$
P(G, x)=\sum_{i=1}^{n}\left(\sum_{j=0}^{n}(-1)^{j} N(i, j)\right) x^{i},
$$

where $N(i, j)$ is the number of spanning subgraphs of $G$ having $i$ components and $j$ edges.

Theorem 2.4 [4] Let $G$ and $H$ be two chromatically equivalent graphs then

- $|V(G)|=|V(H)|,|E(G)|=|E(H)|$. 
- $\chi(G)=\chi(H)$.

- $t_{1}(G)=t_{1}(H)$.

- $t_{2}(G)-2 t_{3}(G)=t_{2}(H)-2 t_{3}(H)$.

- $G$ is connected if and only if $H$ is connected.

- $G$ is 2-connected if and only if $H$ is 2-connected.

- $G$ and $H$ have the same number of shortest cycles; i.e. $g(G)=g(H)$.

Theorem 2.5 [5] Let $G$ and $H$ be chromatically equivalent graphs. If $G$ and $H$ do not contain $K_{4}$ then they have the same number of pure $C_{4}$.

Theorem 2.6 [3] Let $G$ be a graph containing at least two triangles. If there is a vertex of a triangle having degree two in $G$, then $(x-2)^{2}$ divides $P(G, x)$.

\section{Main results}

In this section, chromatic polynomial for necklace graph $N_{n}(n \geq 2)$ is determined. It is further shown that the graph $N_{3}$ is chromatically unique. By using Fundamental reduction theorem, one can easily deduce that

$$
P\left(N_{2}, x\right)=x^{6}-9 x^{5}+34 x^{4}-67 x^{3}+67 x^{2}-26 x .
$$

Theorem 3.1 For $n \geq 3$,

$$
\begin{aligned}
P\left(N_{n}, x\right)= & \left\{(x-2)^{3}-1\right\} x(x-1)(x-2)\left(x^{2}-3 x+3\right)^{n-2}-2 x(1-x)^{n-1}(x-2)^{2} \\
& \left.+2 \sum_{j=2}^{n-1}(1-x)^{n-(j+1)}\left\{P\left(N_{j}, x\right)+x(x-1)(x-2)\left(x^{2}-3 x+3\right)^{j-1}\right)\right\} .
\end{aligned}
$$

Proof: By using Fundamental reduction theorem for $n \geq 3$ and $0 \leq j \leq$ $n-3$, we get

$$
P\left(N_{n-j}, x\right)=(x-2) P\left(L_{n-j}, x\right)-P\left(G_{n-j}, x\right) .
$$

The graph $L_{n-j}$ is a polygone graph consisting of one $C_{3}$ and $(n-j-1) C_{4}$ cycles. The graph $G_{n-j}$ is obtained by contracting the edge $e$ of the necklace graph $N_{n-j}$ (see Figure 2). By using FRT recursively, one can deduce that:

$$
P\left(L_{n-j}, x\right)=x(x-1)(x-2)\left(x^{2}-3 x+3\right)^{n-j-1} .
$$



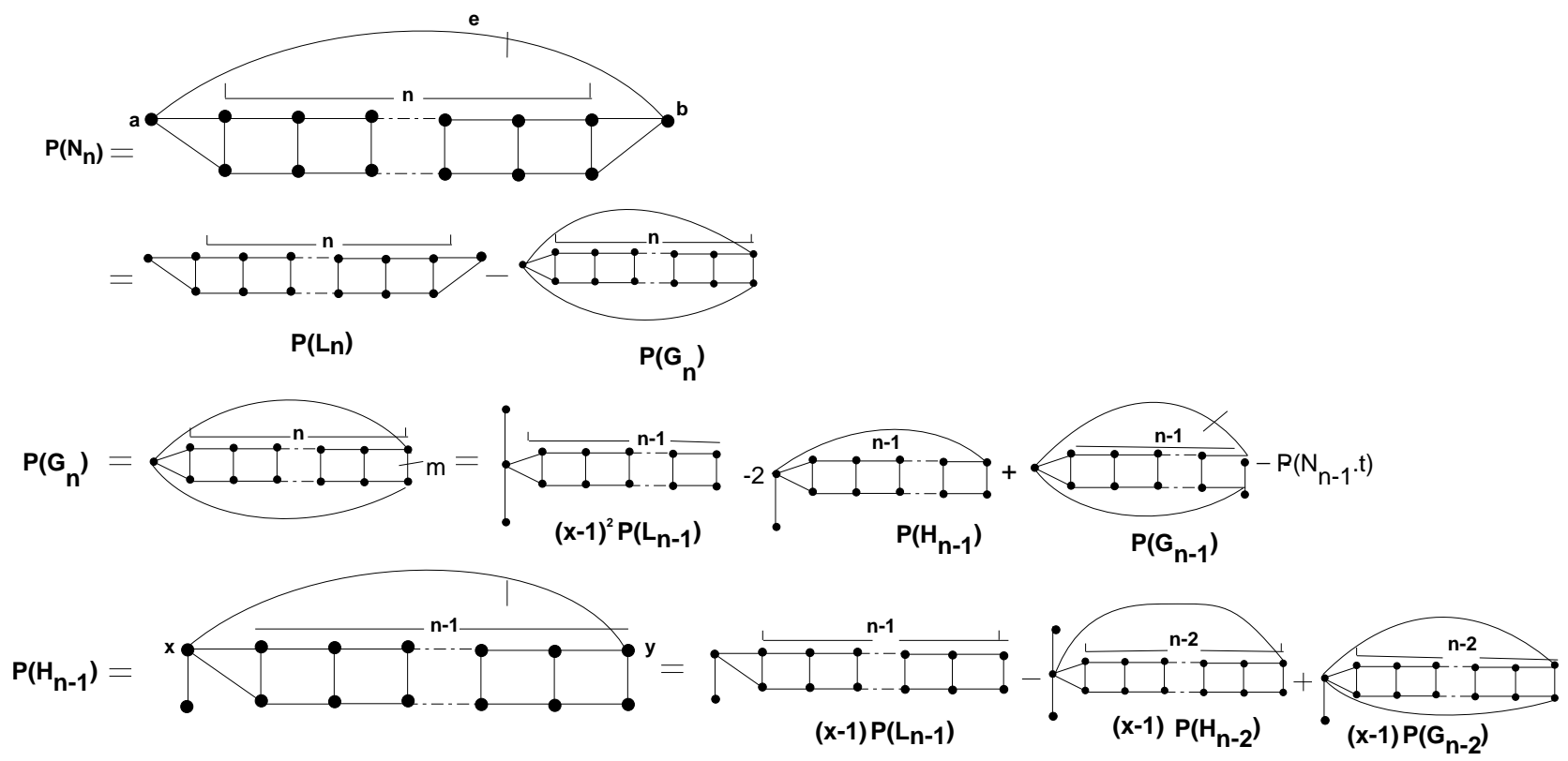

Figure 2: Reduction algorithm on $N_{n}$ graph

$P\left(G_{n-j}, x\right)=(x-1)^{2} P\left(L_{n-j-1}, x\right)-2 P\left(H_{n-j-1}, x\right)+P\left(G_{n-j-1}, x\right)-P\left(N_{n-j-1}, x\right)$.

Also from Figure 2, the chromatic polynomial of the graph $H_{n}$ can be computed and is:

$$
P\left(H_{n}, x\right)=(x-1) P\left(L_{n}, x\right)-(x-1) P\left(H_{n-1}, x\right)+(x-1) P\left(G_{n-1}, x\right) .
$$

Substituting the value of $P\left(G_{n-1}, x\right)$ from 1 in (4), we get

$P\left(H_{n}, x\right)=(x-1) P\left(L_{n}, x\right)-(x-1) P\left(H_{n-1}, x\right)+(x-1)(x-2) P\left(L_{n-1}, x\right)-(x-1) P\left(N_{n-1}, x\right)$.

Similarly

$P\left(H_{n-1}, x\right)=(x-1) P\left(L_{n-1}, x\right)-(x-1) P\left(H_{n-2}, x\right)+(x-1)(x-2) P\left(L_{n-2}, x\right)-(x-1) P\left(N_{n-2}, x\right)$,

$P\left(H_{n-2}, x\right)=(x-1) P\left(L_{n-2}, x\right)-(x-1) P\left(H_{n-3}, x\right)+(x-1)(x-2) P\left(L_{n-3}, x\right)-(x-1) P\left(N_{n-3}, x\right)$,

continuing in this way we have

$P\left(H_{3}, x\right)=(x-1) P\left(L_{3}, x\right)-(x-1) P\left(H_{2}, x\right)+(x-1)(x-2) P\left(L_{2}, x\right)-(x-1) P\left(N_{2}, x\right)$.

By replacing these values recursively in equations (3) for $j=0$, we get $P\left(G_{n}, x\right)=\left(x^{2}-3 x+3\right) P\left(L_{n-1}, x\right)-2 \Sigma_{j=2}^{n-1}(x-1)^{n-(j+1)}(-1)^{n-(j+1)}\left\{P\left(N_{j}, x\right)\right.$ 


$$
\left.+P\left(L_{j}, x\right)\right\}+(-1)^{n-3}(x-1)^{(n-3)}\left\{P\left(H_{2}, x\right)-(x-1) P\left(L_{2}, x\right)\right\} .
$$

Since the chromatic polynomial of the necklace graph $N_{n}$ is

$$
P\left(N_{n}, x\right)=(x-2) P\left(L_{n}, x\right)-P\left(G_{n}, x\right) .
$$

Therefore by replacing the values of $P\left(G_{n}, x\right)$ and $P\left(L_{n}, x\right)$ in the equation (6) we conclude that

$$
\begin{aligned}
P\left(N_{n}, x\right)= & \left\{(x-2)^{3}-1\right\} x(x-1)(x-2)\left(x^{2}-3 x+3\right)^{n-2}-2 x(1-x)^{n-1}(x-2)^{2} \\
& \left.+2 \sum_{j=2}^{n-1}(1-x)^{n-(j+1)}\left\{P\left(N_{j}, x\right)+x(x-1)(x-2)\left(x^{2}-3 x+3\right)^{j-1}\right)\right\} .
\end{aligned}
$$

In the next theorem, the chromatic uniqueness of the necklace graph $N_{3}$ is established which is also the main result of this paper.

Theorem 3.2 Necklace graph $N_{3}$ is chromatically unique.

Proof: Suppose $N_{3}$ is not chromatically unique and there is another graph $H$ which has the same chromatic polynomial as $N_{3}$. Then

$P\left(N_{3}\right)=P(H, x)=x^{8}-12 x^{7}+64 x^{6}-198 x^{5}+386 x^{4}-474 x^{3}+321 x^{2}-74 x$.

Then by Theorem 2.4 we have following information about $H$.

1. $H$ has 8 vertices and 12 edges. Also $t_{1}(H)=2$, i.e. $H$ has exactly 2 triangles.

2. Since there is no $K_{4}$ in $H$ therefore by Theorem $2.5 t_{2}(H)=2$.

3. $H$ contain no separating(cut) vertex as $H$ is 2-connected graph.

4. $H$ does not contain any vertex of degree 2 that lies in a triangle. Since otherwise $(x-2)^{2} \mid P(H, x)$ by Theorem 2.6 .

5. By Theorem 2.2, the coefficient $a_{4}$ of the $P(H, x)$ is

$$
\begin{aligned}
& a_{4}=\left(\begin{array}{c}
m \\
4
\end{array}\right)-\left(\begin{array}{c}
m-2 \\
2
\end{array}\right) t_{1}(G)-\left(\begin{array}{c}
t_{1}(G) \\
2
\end{array}\right)-(m-3) t_{2}(G)+(2 m- \\
& 9) t_{3}(G)-t_{4}(G)+6 t_{5}(G)+t_{6}(G)+2 t_{7}(G)+3 t_{8}(G) .
\end{aligned}
$$

One can deduce that

$$
a_{4}=-200+t_{4}(H)-2 t_{7}(H) .
$$


By comparing the coefficient of $x_{5}$ in (7) and $a_{4}$, we get

$$
t_{4}(H)-2 t_{7}(H)=2 .
$$

Since $t_{7}(H)<2$, otherwise number of triangles increased by 2 . So we have two subcases

(a) $t_{7}(H)=1 \Rightarrow t_{4}(H)=4$

(b) $t_{7}(H)=0 \Rightarrow t_{4}(H)=2$.

Since $H$ has exactly two triangles. Let $k$ be the number of vertices covered by these two triangles then degree of each vertex of triangle must be $\geq 3$, therefore remaining $n-k$ vertices of $H$ has at least degree 2. By Hand Shaking Lemma we have

$$
2(n+4)=2 n+8 \geq 3 k+2(n-k)=3 k+2 n-2 k .
$$

This implies that $k \leq 8$ and clearly $k \neq 3,7,8$. Hence possible values of $k$ are 4,5 and 6 . Therefore $H$ must have one of the subgraph $G_{1}$, or $G_{2}$ or $G_{3}$. These subgraphs are shown in the Figure 3.

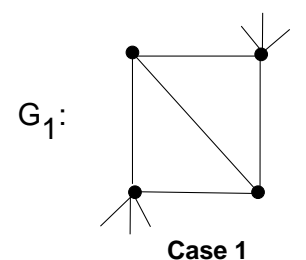

Case 1

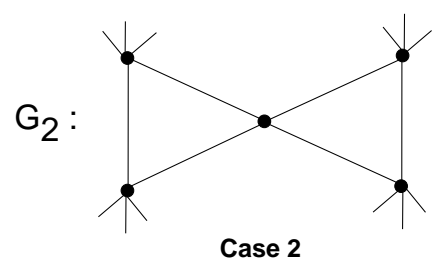

Case 2
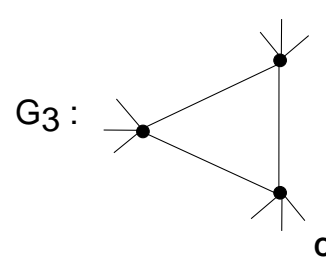

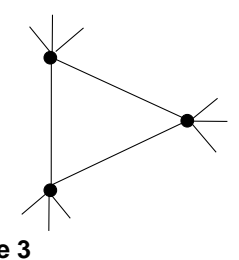

Figure 3: Possible subgraphs of $H$

Case 1 If $k=4$ then the two triangles cover 4 vertices. In this case $H$ has a subgraph $G_{1}$ which is edge gluing of two triangles as shown in the Figure 3. From above information, there are three possible subgraphs of $H$ in this case as shown in the Figure 4. We prove that $H$ can not have any of these subgraph.

Subcase 1.1 In subgraph (I), $x$ and $y$ are vertices of triangle so $d(x)$ and $d(y) \geq 3$. This implies that at most one of $x$ and $y$ can be adjacent to the vertex $b$ otherwise there exist at least 3 triangles, a contradiction.

Also both $x$ and $y$ are not connected to the vertex $b$ otherwise there exists at least three pure $C_{4}$ cycles. So without loss of generality, suppose that there is an edge between vertex $x$ and $b$ and there is a path of length 2 from $y$ to 
(I)

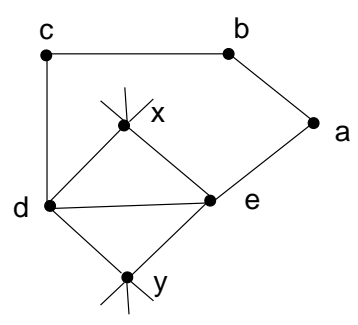

(II)

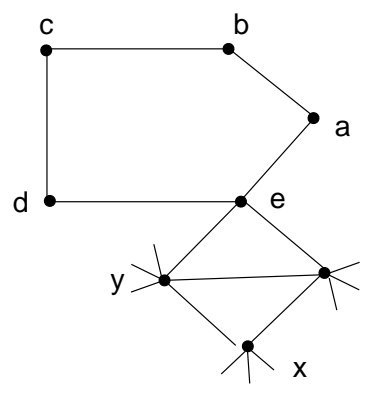

(IIII)

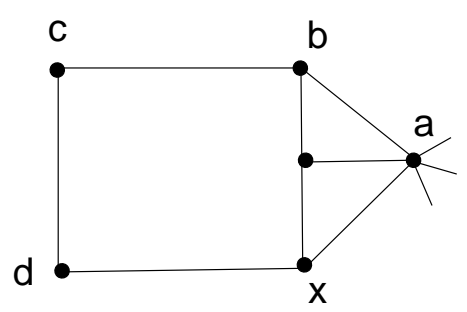

Figure 4: Possible subgraphs in Case (1)

any other vertex. But again in this case at least 3 pure $C_{4}$ cycles exists, a contradiction. Hence, $x$ and $y$ are not adjacent to vertex $b$.

Since $H$ has 8 vertices, so there must be a path of length two from $x$ to $y$. Let $z$ be a vertex between $x$ and $y$, then the graph $H$ has the form shown in the Figure 5. In this case, the vertex $z$ can only be adjacent to $b$
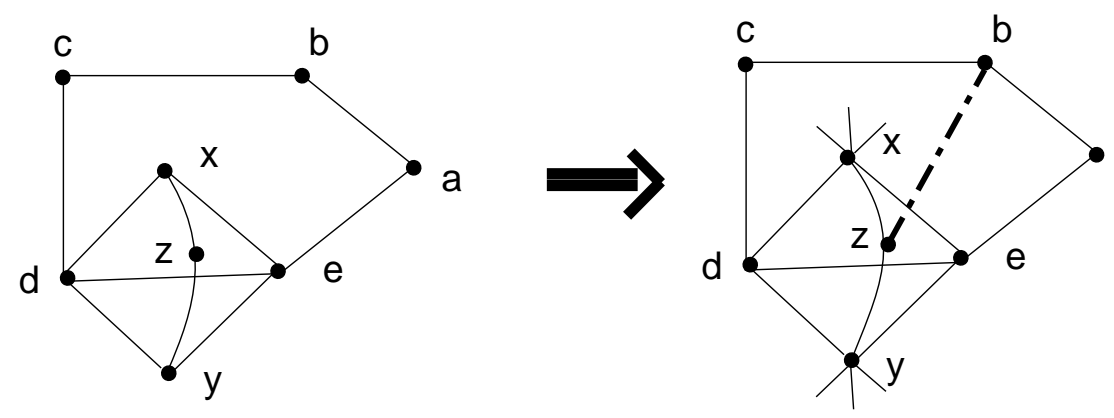

Figure 5: Subgraph(I)

otherwise pure $C_{4}>2$. But if $z$ is adjacent to $b$ then again $t_{4}(H)=3$ (as $t_{7}(H)=0 \Rightarrow t_{4}(H)=2$ ), a contradiction.

Subcase 1.2 Since $x$ is a vertex of triangle, so $d(x) \geq 3$. This implies that there must be an edge from $x$ to any other vertex of $H$. Therefore, the subgraph (II) can be extended to two possible graphs shown in the Figure 6.

- If $y$ is adjacent to $c$ then $n\left(t_{4}(H)\right)=4$, a contradiction. Because $t_{7}(H)=0 \Rightarrow t_{4}(H)=2$. 
(a)

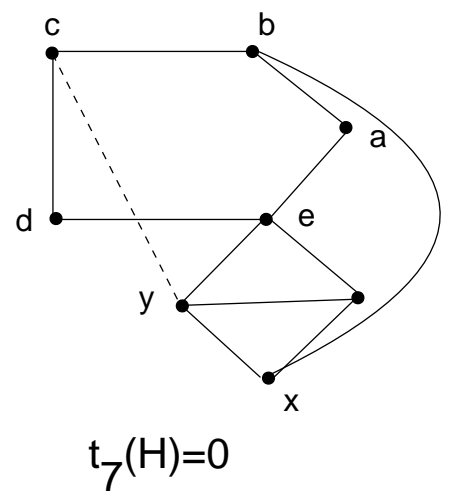

(b)

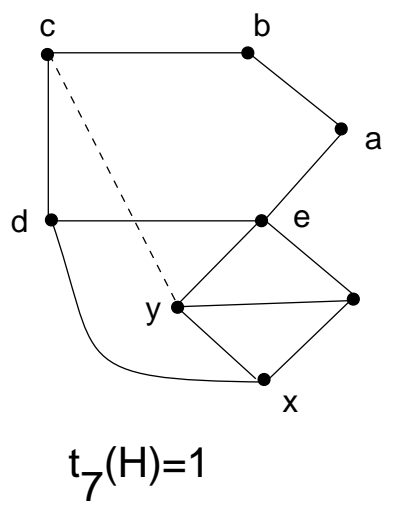

Figure 6: Subcases of subgraph(II)

- If $y$ is adjacent to $c$ then $t_{4}(H)=2$ a contradiction. Because $t_{7}(H)=$ $1 \Rightarrow t_{4}(H)=4$.

Subcase 1.3 There are 6 vertices and 8 edges in this subgraph, so two more vertices and four edges needed to be included. So either there exists a path of leng th three between any two vertices. or there are two paths of length two between any two vertices. Both of these situations are shown in the Figure 7. In the first situation, suppose there exist a path of length 3 between the

(a)

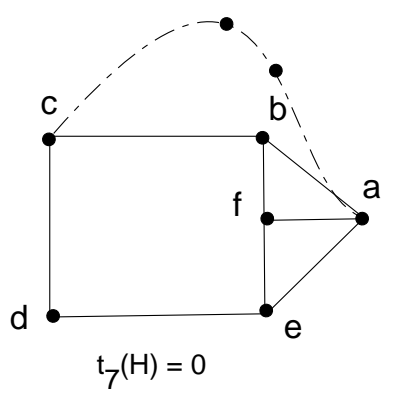

(b)

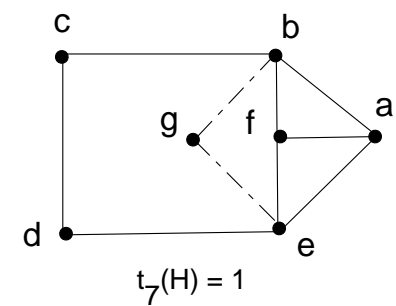

Figure 7: Possibilities in subgraph (III)

vertices $a$ and $c$. But in this case $t_{4}(H)>3$, a contradiction. Since in this case $t_{7}(H)=0 \Rightarrow t_{4}(H)=2$. 
If the second situation arises then there must exist at least one path of length 2 from vertex $b$ to $e$ as shown in the Figure 7. Otherwise $t_{2}(H)>3$. Let $g$ be a new vertex in $b-e$ path, then this new graph has two pure $C_{4}$ and a 5 -vertex wheel graph with one spoke deleted. Since, one more path of length 2 has to be added. But by adding such path leads to a third pure $C_{4}$, a contradiction.

Hence $H$ does not contain any of the subgraphs (I), (II) and (III). This implies that $H$ does not contain the subgraph $G_{1}$ which is the edge gluing of 2-triangles.

Case 2 In this case $H$ contain the subgraph $G_{2}$. Since $G_{2}$ has a cut vertex and two triangles, therefore there should be a path of length at least 2 between any two distinct vertices of 2 triangles. Also $H$ has 8 vertices so we needed to add 3 more vertices in $G_{2}$, therefore the length of this path is at most 4. So $G_{2}$ can extended to the three subgraphs $G_{21}, G_{22}$ and $G_{33}$ shown in Figure 8. Subcase 2.1: Let $x$ and $y$ are vertices of the triangles then $d(x), d(y) \geq 3$.

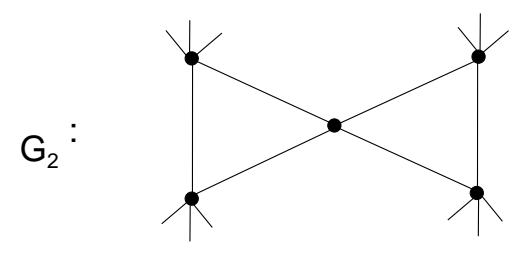

Case (2) when $\mathrm{K}=5$
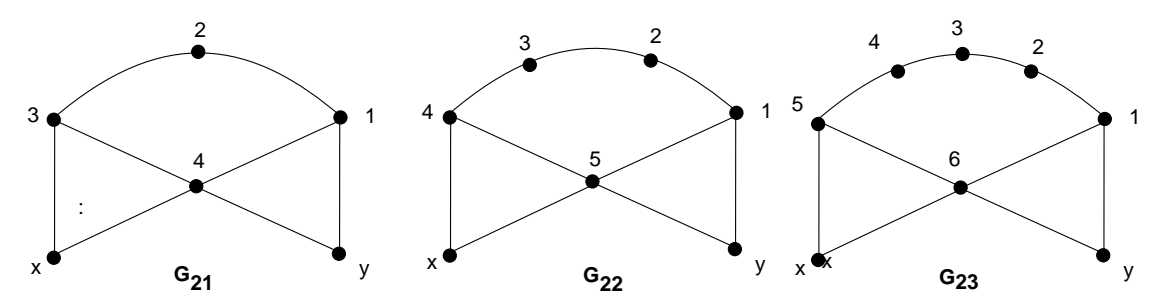

Subcases of Case(2)

Figure 8: Case(2) of subgraph $\mathrm{H}$

$x$ and $y$ can not be adjacent to vertex 2 otherwise $t_{1}\left(G_{21}\right)>2$. So we have following three possible extension for the subgraph $G_{21}$.

1. There is a path of length two between the vertices $x$ and $y$. Let $z$ be a vertex added to the subgraph $G_{21}$. Since $t_{2}(H)=2$, therefore there 
must be a path of length two from vertex $z$ to vertex 2 . So the only possible graph in this case is $G_{211}$ shown in the Figure 9.

But $P\left(G_{211}, x\right)=x^{8}-12 x^{7}+64 x^{6}-192 x^{5}+398 x^{4}-495 x^{3}+358 x^{2}-$ $112 x \neq P\left(N_{3}, x\right)$.

2. There are two paths of length two from the vertices $x$ and $y$ to the vertex 2 . Let $G_{212}$ be the new graph (see Figure 9 ) formed by adding these two paths. Then, clearly $t_{2}\left(G_{212}\right) \geq 3$, a contradiction.

3. There is a path of length three from vertices $x$ or $y$ to any other vertex. Suppose there is a path of length 3 from vertex $y$ to vertex 1 . Let $G_{213}$ be the graph obtained by adding this path. Since $d(x) \geq 3$ and $x$ can be adjacent only to the vertex 5 or 6 but in either cases we have at least 3 pure $C_{4}$, a contradiction.

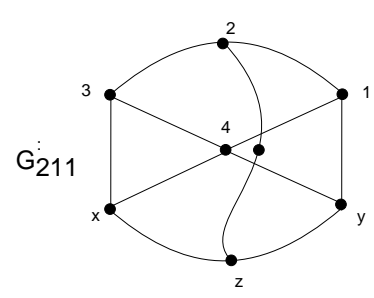

A path of lenght 2 $b / w$ vertices $x$ and $y$

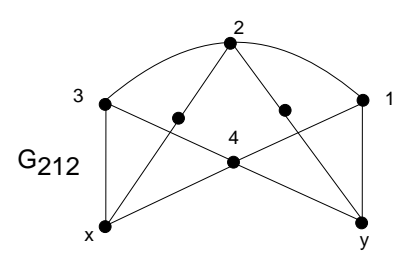

Two paths of lenght 2

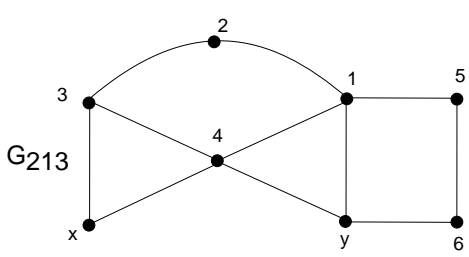

A path of lenght 3 $\mathrm{b} / \mathrm{w}$ vertices $\mathrm{y}$ and 1

Figure 9: Possible subgraphs in subcase 2.1

Subcase 2.2: Suppose $H$ contain the subgraph $G_{22}$. Since $d(x), d(y) \geq 3$, so there are following two possibilities:

1. There is a path of length two between the vertices $x$ and $y$. Since one more edge is needed to be added. But by adding this edge, the resulting graph has at least 3 pure $C_{4}$, a contradiction.

2. There is a path of length two from the vertex $x$ or $y$ to any vertex of graph $G_{22}$. Suppose this path is from the vertex $x$ to the vertex 3. Since $d(y) \geq 3$ and $y$ can only be adjacent to vertex 3 or $z$, otherwise number of triangles exceed from 2. Both these constructions gives at least 3 pure $C_{5}$ and no 5 -vertex wheels with one spoke deleted, a contradiction. Since $t_{7}(H)=0 \rightarrow t_{4}(H)=2$. contradiction.

The graphs constructed in this case are shown in Figure 10. 

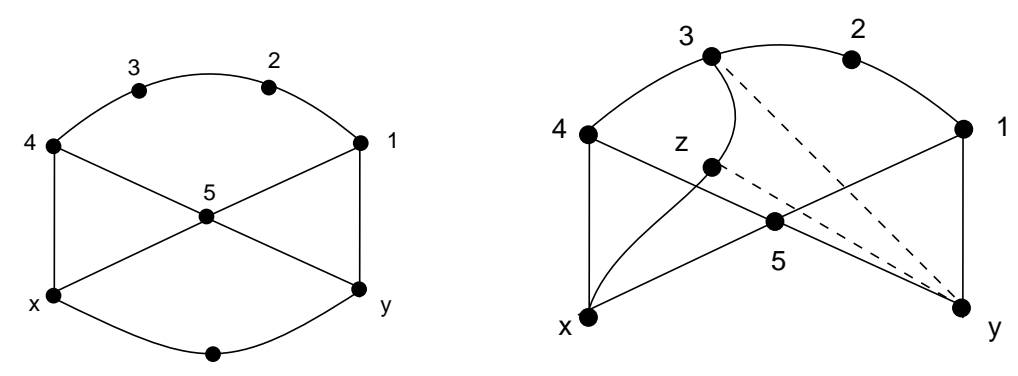

Figure 10: Possible subgraphs in subcase 2.2

Subcase 2.3: Suppose $H$ contain the subgraph $G_{23}$. Again since $d(x), d(y) \geq$ 3 , we have the following three possible extensions of the subgraph $G_{23}$.

1. Suppose $x$ and $y$ are adjacent to the vertices $a$ and $b$ respectively (See Figure 11). But in this construction $t_{4}(H) \geq 3$ and $t_{7}(h)=0$, a contradiction, since $t_{7}(H)=0 \rightarrow t_{4}(H)=2$.

2. if the vertices $x$ and $y$ are adjacent to the vertex $b$. Again, in this construction $t_{4}(H) \geq 3$ and $t_{7}(h)=0$, a contradiction, since $t_{7}(H)=$ $0 \rightarrow t_{4}(H)=2$.

3. $x$ and $y$ adjacent to the vertices $a$ and $c$, respectively. The graph obtained in this case is denoted by $G_{233}$ (see the Figure 11).

But $P\left(G_{233}, x\right)=x^{8}-12 x^{7}+64 x^{6}-197 x^{5}+376 x^{4}-440 x_{3}+288 x^{2}-80 x \neq$ $P(H, x)$.

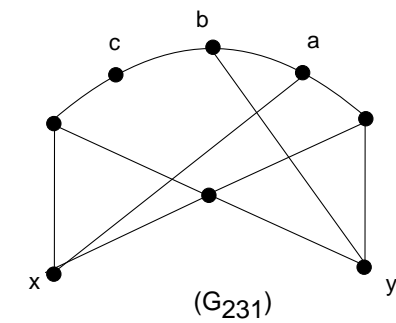

$\left(G_{231}\right)$

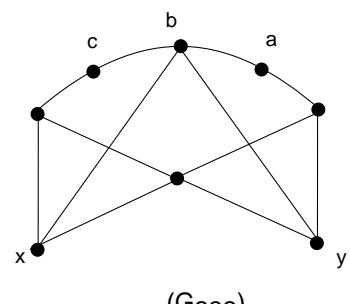

$\left(G_{232}\right)$

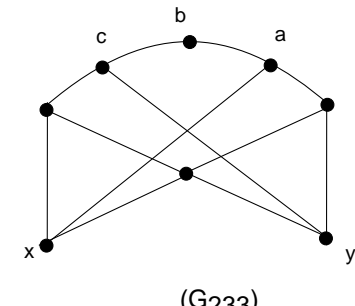

$\left(\mathrm{G}_{233}\right)$

Figure 11: Possible subgraphs in Subcases 2.3

Summing up all above subcases, we conclude that $H$ does not contain the subgraph $G_{2}$. 
Case 3: In this case $H$ contain the subgraph $G_{3}$ which has exactly two disjoint triangles. So 6 vertices required to cover two disjoint triangles. Furthermore $t_{2}(H)=2$ implies that there are following subcases:

Subcase 3.1 In this subcase there are two disjoint $C_{4}$ cycles. Since we have two disjoint triangles therefore $t_{7}(H)=0$ and this implies that $t_{4}(H)=2$. All possible subgraph in this situation are shown in Figure 12. But in each of these subgraphs $t_{4}(H) \neq 2$.

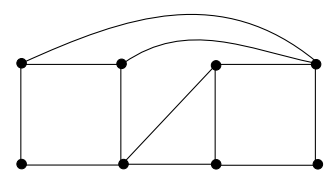

(1)

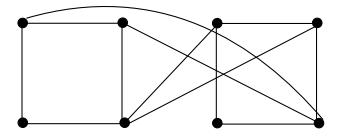

(4)

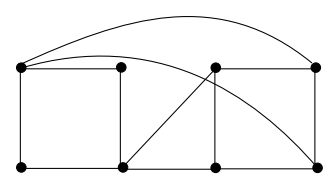

(2)

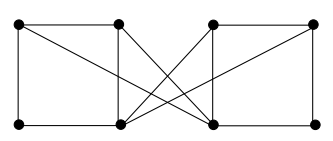

(5)

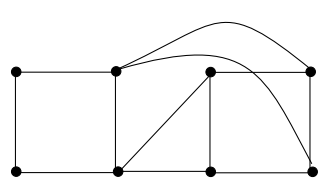

(3)

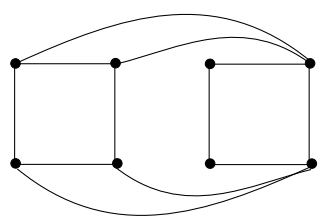

(6)

Figure 12: List of subgraphs in subcase 3.1

Subcase (3.2) If the graph $G_{3}$ contain two pure $C_{4}$ with one vertex in common then the only non-isomorphic graph obtained in this case has at least three pure $C_{5}$ and is shown in Figure 13 .

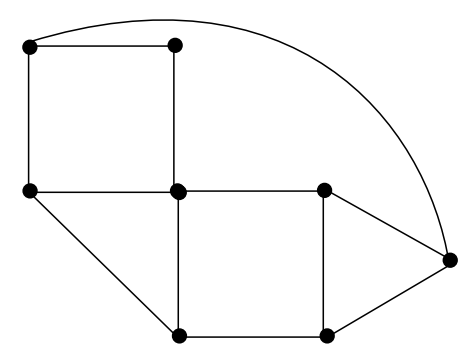

Figure 13: Graph in subcase 3.2

Subcase 3.3 If the graph $G_{3}$ contains two pure $C_{4}$ cycles having a path $P_{3}$ 
in common. The construction of subgraphs in this case leads to contradiction except the subgraph $G_{334}$ (shown in Figure 14). As $d(x), d(y) \geq 3$ therefore
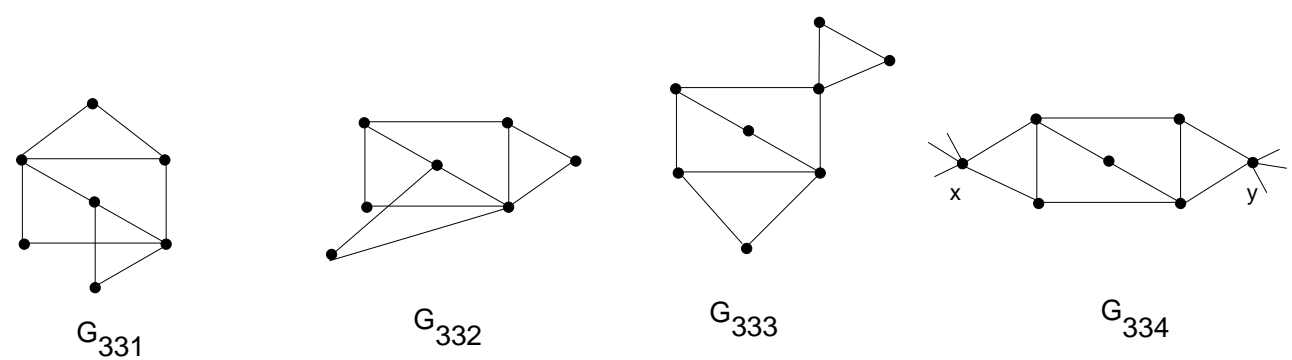

Figure 14: Graph in subcase (3.3)

there is a path of length 2 between vertex $x$ and $y$. This construction leads to a subgraph $G_{334}$ (shown in Figure 15).

But $P\left(G_{334}, x\right)=x^{8}-12 x^{7}+64 x^{6}-197 x^{5}+387 x^{4}-450 x^{3}+304 x^{2}-88 x \neq$ $P(H, x)$. Subcase 3.4 If the graph $G_{3}$ contain two pure $C_{4}$ having one edge

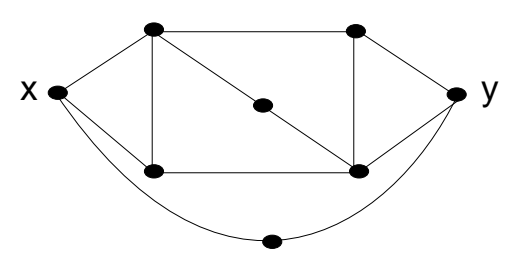

Figure 15: Subgraph $G_{334}$

in common then the four subgraphs obtained in this case are shown in the Figure 16.

Since $d(x), d(y) \geq 3$ so there must be an edge between these two vertices. But by adding this edge in each of the graphs $G_{341}, G_{342}$ and $G_{343}$ implies $t_{2}(H) \geq 3$. If we add the edge between the vertex $x$ and $y$ of the graph $G_{344}$, the resulting graph will become isomorphic to $N_{3}$.

Hence the necklace graph $N_{3}$ is chromatically unique.

We state the following open problem.

\section{Open Problem}

Investigate the chromatic uniqueness of necklace graph $N_{n}$ for $n \geq 4$. 


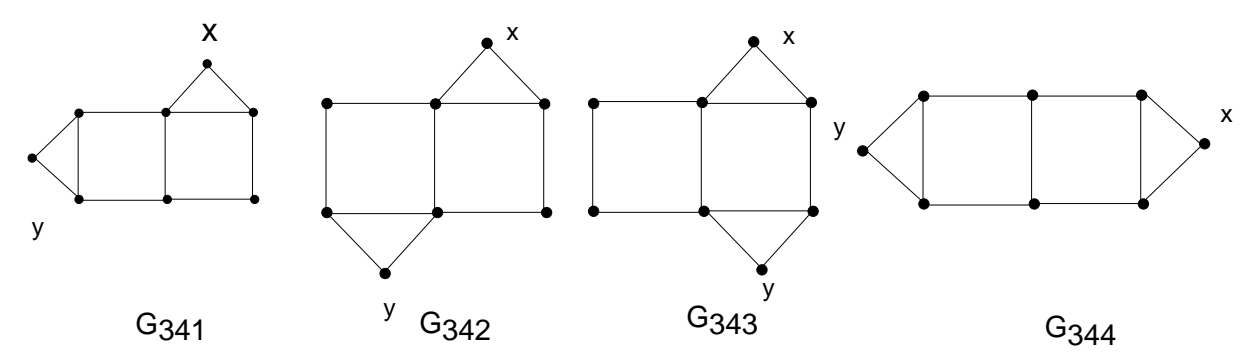

Figure 16: Graphs in subcase (3.4)

\section{References}

[1] G. D. Birkhoff, A determinant formula for the number of ways of colouring a map, Annl. of Math. 14, no. 2, (1912), 42-46.

[2] C. Y. Chao, E. G. Whitehead, Jr., On chromatic equivalence of graphs, in Y. Alavi and D. R. Lick, (Eds.) Theory and Applications of Graphs, Lecture Notes in Math., Vol. 642, Springer, New York, Berlin, 1978. 121-131.

[3] C. Y. Chao, E. G. Whitehead Jr., Chromatically unique graphs, Discrete Math., 27 (1979) 171-177.

[4] K. M. Koh and K. L. Teo, The search for chromatically unique graphs, Disc. Math. 172 (1997) 59-72..

[5] K. M. Koh, K. L. Teo, Chromatic classes of 2-connected (n; n + 3)graphs with at least two triangles, Disc. Math. 127 (1994) 243258.

[6] L. Lovasz, M. D. Plummer, On a family of planar bicritical //graphs, Proc. London Math. Soc., 30 (1975),160-176.

[7] K. L. Teo, K. M. Koh, Chromatic classes of certain 2-connected (n; n+2)-graphs, Ars Combin., 32 (1991) 6576.

[8] H. Whitney, A logical expansion in mathematics, Bull. Amer. Math. Soc., 38, 572-579(1932).

[9] H. Whitney, The coloring of graphs, Ann. Math., 33 (1932) 688718. 